\title{
An expectancy model of Chinese-American differences in conflict-avoiding
}

Ray Friedman ${ }^{1}$, Shu-Cheng

$\mathrm{Chi}^{2}$ and Leigh Anne $\mathrm{Liu}^{3}$

${ }^{1}$ Owen Graduate School of Management, Vanderbilt University, Nashville, TN, USA;

${ }^{2}$ Department of Business Administration, National Taiwan University, Taipei, Taiwan; ${ }^{3}$ Robinson College of Business, Georgia State University, Atlanta, GA, USA
Journal of International Business Studies (2006), doi: I0. I057/palgrave.jibs.84002 I2

Correction to: Journal of International Business Studies (2006) 37(1), 76-91. doi:10.1057/palgrave.jibs.8400172

Due to a processing error, some values in Tables 2 and 3 of the above paper were reproduced incorrectly. The correct versions of the tables are reproduced below.

The authors are grateful to Phoebus Lee for pointing out this error.

Table 2 Regression models

\begin{tabular}{|c|c|c|c|c|c|c|c|c|}
\hline Dependent variables & Avoiding & $\begin{array}{c}\text { Hurt } \\
\text { relationship }\end{array}$ & $\begin{array}{c}\text { Relationship } \\
\text { payoff }\end{array}$ & $\begin{array}{l}\text { Concern } \\
\text { for others }\end{array}$ & Avoiding & Avoiding & Avoiding & Avoiding \\
\hline Model & (1) & (2) & (3) & (4) & (5) & (6) & (7) & (8) \\
\hline Hypothesis tested & $\mathrm{H} 1$ & $\mathrm{H} 2$ & $\mathrm{H} 4$ & $\mathrm{H} 6$ & $\mathrm{H} 3$ & $\mathrm{H} 5$ & $\mathrm{H7}$ & - \\
\hline Overall $F$ & 33.95 & 7.23 & 4.35 & 16.49 & 53.94 & 47.05 & 51.56 & 46.47 \\
\hline Model $R^{2}$ adj. & 0.40 & 0.15 & 0.06 & 0.24 & 0.49 & 0.43 & 0.47 & 0.56 \\
\hline d.f. & 6,290 & 6,290 & 6,290 & 6,290 & 7,289 & 7,289 & 7,289 & 9,287 \\
\hline Variables & $\beta$ & $\beta$ & $\beta$ & $\beta$ & $\beta$ & $\beta$ & $\beta$ & $\beta$ \\
\hline \multicolumn{9}{|l|}{ Controls } \\
\hline Age & -0.05 & -0.07 & 0.12 & 0.06 & -0.02 & -0.07 & -0.06 & -0.05 \\
\hline Sex & -0.04 & 0.02 & 0.02 & 0.00 & -0.05 & -0.04 & -0.04 & -0.05 \\
\hline Experience & $0.15^{*}$ & 0.07 & -0.09 & -0.07 & $0.12^{*}$ & $0.16^{* *}$ & $0.17^{\star *}$ & $0.15^{\star *}$ \\
\hline Rank & -0.12 & 0.04 & $0.16^{*}$ & -0.07 & $-0.14^{\star \star}$ & $-0.15^{\star *}$ & $-0.10^{*}$ & $-0.12^{\star \star}$ \\
\hline Upset & $-0.20^{\star \star}$ & $0.17^{* * *}$ & $0.24^{\star * *}$ & $-0.27^{\star \star *}$ & $-0.26^{* * *}$ & $-0.25^{\star \star *}$ & $-0.12^{\star *}$ & $-0.19^{* * *}$ \\
\hline Chinese $($ American $=0$, Chinese $=1$ ) & $0.53^{* * *}$ & $0.37^{* * *}$ & 0.08 & $0.38^{\star \star \star}$ & $0.41^{* * *}$ & $0.51^{* * *}$ & $0.41^{* * *}$ & $0.32^{\star \star *}$ \\
\hline \multicolumn{9}{|l|}{ Expectancy model predictors } \\
\hline Hurt relationship & & & & & $0.32^{* * *}$ & & & $0.27^{* * *}$ \\
\hline Relationship payoff & & & & & & $0.17^{\star \star *}$ & & 0.05 \\
\hline Concern for others & & & & & & & $0.30^{* * *}$ & $0.27 * * *$ \\
\hline
\end{tabular}

${ }^{*} P<0.05,{ }^{* *} P<0.01,{ }^{* * *} P<0.001$ (all two-tailed). 
Table 3 Regression models for interaction effects

\begin{tabular}{|c|c|c|c|c|c|}
\hline Dependent variables & Relationship payoff & Hurt relationship & Avoiding & Avoiding & Avoiding \\
\hline Model & (1) & (2) & (3) & (4) & (5) \\
\hline Hypothesis tested & $\mathrm{H} 8$ & $\mathrm{H} 8$ & $\mathrm{H} 9$ & $\mathrm{H} 10$ & $\mathrm{H} 10$ \\
\hline Overall $F$ & 5.53 & 8.17 & 27.00 & 25.78 & 32.78 \\
\hline Model $R^{2}$ adj. & 0.11 & 0.16 & 0.41 & 0.43 & 0.49 \\
\hline d.f. & 8,288 & 8,288 & 8,288 & 9,287 & 9,287 \\
\hline Variables & $\beta$ & $\beta$ & $\beta$ & $\beta$ & $\beta$ \\
\hline \multicolumn{6}{|l|}{ Controls } \\
\hline Age & 0.11 & -0.08 & -0.05 & -0.07 & -0.03 \\
\hline Sex & 0.05 & 0.04 & -0.03 & -0.03 & -0.04 \\
\hline Experience & -0.09 & 0.07 & $0.15^{*}$ & $0.16^{\star *}$ & $0.13^{*}$ \\
\hline Rank & $0.16^{*}$ & 0.04 & $-0.12^{\star *}$ & $-0.15^{\star \star}$ & $-0.13^{\star *}$ \\
\hline Upset & $0.23^{* * *}$ & $0.17^{* *}$ & $-0.21^{* * *}$ & $-0.25^{* * *}$ & $-0.26^{\star * *}$ \\
\hline Chinese $($ American $=0$, Chinese $=1$ ) & 0.27 & $0.86^{* * *}$ & $0.78^{* * *}$ & $0.74^{\star * *}$ & $0.51^{* * *}$ \\
\hline Boss & $-0.28^{* * *}$ & $-0.25^{\star * *}$ & $-0.17^{\star \star}$ & $-0.13^{*}$ & -0.09 \\
\hline Chinese $\times$ boss & 0.21 & $0.55^{\star *}$ & $0.28^{*}$ & $0.25^{*}$ & 0.11 \\
\hline Relationship payoff & & & & $0.15^{* *}$ & \\
\hline Hurt relationship & & & & & $0.31^{* \star *}$ \\
\hline
\end{tabular}

${ }^{*} P<0.05,{ }^{* *} P<0.01,{ }^{* *} P<0.001$ (all two-tailed). 